\title{
Skeletal muscle-specific Cre recombinase expression, controlled by the human a-skeletal actin promoter, improves glucose tolerance in mice fed a high-fat diet
}

\author{
Rami Al Batran ${ }^{1,2,3}$ - Keshav Gopal ${ }^{1,2,3}$ - Mackenzie D. Martin ${ }^{4} \cdot K_{\text {Kim L. Ho }}^{1,2,3}$ • Malak Almutairi ${ }^{1,2,3}$ • \\ Hanin Aburasayn ${ }^{1,2,3}$. Farah Eaton ${ }^{1,2,3}$ • Jonathan E. Campbell ${ }^{4}$. John R. Ussher ${ }^{1,2,3}$
}

Received: 16 February 2018 / Accepted: 19 April 2018 / Published online: 1 June 2018

(C) Springer-Verlag GmbH Germany, part of Springer Nature 2018

\begin{abstract}
Aims/hypothesis Cre-loxP systems are frequently used in mouse genetics as research tools for studying tissue-specific functions of numerous genes/proteins. However, the expression of Cre recombinase in a tissue-specific manner often produces undesirable changes in mouse biology that can confound data interpretation when using these tools to generate tissue-specific gene knockout mice. Our objective was to characterise the actions of Cre recombinase in skeletal muscle, and we anticipated that skeletal muscle-specific Cre recombinase expression driven by the human $\alpha$-skeletal actin (HSA) promoter would influence glucose homeostasis.

Methods Eight-week-old HSA-Cre expressing mice and their wild-type littermates were fed a low- or high-fat diet for 12 weeks. Glucose homeostasis (glucose/insulin tolerance testing) and whole-body energy metabolism (indirect calorimetry) were assessed. We also measured circulating insulin levels and the muscle expression of key regulators of energy metabolism.

Results Whereas tamoxifen-treated HSA-Cre mice fed a low-fat diet exhibited no alterations in glucose homeostasis, we observed marked improvements in glucose tolerance in tamoxifen-treated, but not corn-oil-treated, HSA-Cre mice fed a high-fat diet vs their wild-type littermates. Moreover, Cre dissociation from heat shock protein 90 and translocation to the nucleus was only seen following tamoxifen treatment. These improvements in glucose tolerance were not due to improvements in insulin sensitivity/signalling or enhanced energy metabolism, but appeared to stem from increases in circulating insulin.

Conclusions/interpretation The intrinsic glycaemia phenotype in the HSA-Cre mouse necessitates the use of HSA-Cre controls, treated with tamoxifen, when using Cre-loxP models to investigate skeletal muscle-specific gene/protein function and glucose homeostasis.
\end{abstract}

Keywords Cre recombinase $\cdot$ Glucose tolerance $\cdot$ Insulin $\cdot$ Skeletal muscle

Electronic supplementary material The online version of this article (https://doi.org/10.1007/s00125-018-4643-x) contains peer-reviewed but unedited supplementary material, which is available to authorised users.

John R. Ussher

jussher@ualberta.ca

1 Katz Centre for Pharmacy and Health Research, Faculty of Pharmacy and Pharmaceutical Sciences, University of Alberta,

Edmonton, AB T6G 2E1, Canada

2 Alberta Diabetes Institute, University of Alberta, Edmonton, AB, Canada

3 Mazankowski Alberta Heart Institute, University of Alberta, Edmonton, AB, Canada

4 Duke Molecular Physiology Institute, Duke University, Durham, NC, USA

\begin{tabular}{|c|c|}
\hline \multicolumn{2}{|c|}{ Abbreviations } \\
\hline BSA & Bovine serum albumin \\
\hline $\mathrm{CM}$ & Conditioned media \\
\hline $\mathrm{D}-\mathrm{Ala}^{2} \mathrm{GIP}$ & $\begin{array}{l}\text { D-Ala }{ }^{2} \text { glucose-dependent insulinotropic } \\
\text { polypeptide }\end{array}$ \\
\hline GSIS & Glucose-stimulated insulin secretion \\
\hline GSK & Glycogen synthase kinase \\
\hline HFD & High-fat diet \\
\hline HSA & Human $\alpha$-skeletal actin \\
\hline Hsp & Heat shock protein \\
\hline $\mathrm{KO}$ & Knockout \\
\hline LFD & Low-fat diet \\
\hline MCK & Muscle creatine kinase \\
\hline mTOR & Mammalian target of rapamycin \\
\hline PPAR & Peroxisome proliferator-activated receptor \\
\hline
\end{tabular}




\section{Research in context}

\section{What is already known about this subject?}

- Tissue-specific Cre recombinase expression, which is often used in mouse genetics, may produce an underlying phenotype that could confound data interpretation

\section{What is the key question?}

- Does Cre recombinase expression within skeletal muscle produce underlying changes in mouse biology that can confound data interpretation?

\section{What are the new findings?}

- Cre recombinase expression driven by the tamoxifen-inducible human $\alpha$-skeletal actin (HSA) promoter improves glucose tolerance in mice fed a high-fat diet

- $\quad$ HSA-Cre-expressing mice are therefore the most appropriate control littermate when using this Cre-loxP system to generate skeletal muscle-specific gene knockout mice

\section{How might this impact on clinical practice in the foreseeable future?}

- Our findings will lead to the appropriate control littermates being used for studies investigating skeletal musclespecific regulation of blood glucose in obesity and the metabolic syndrome, possibly leading to higher success rates for translating benchside findings to the bedside

TAG Triacylglycerol

VDAC Voltage-dependent anion-selective channel WT Wild-type

\section{Introduction}

Cre-loxP technology has been widely adopted as a tool for selective cell-targeted manipulation of gene expression [1]. Through the use of the Cre-loxP system in mice, cell/tissuespecific promoters drive Cre recombinase expression in the organ of interest, following which Cre recombinase can excise the floxed gene, allowing for generation of a tissue-specific gene knockout (KO) mouse [1]. In addition, many Cre-loxP systems now employ inducible systems, such as those that are sensitive to tamoxifen but insensitive to endogenous oestrogen, thereby allowing the generation of tissue-specific, temporally controlled gene $\mathrm{KO}$ adult mice. Of importance, we have become increasingly aware that Cre recombinase expression or activity per se can impact tissue function. This can arise from the design of the construct used to drive Cre expression, as certain Cre constructs incorporate additional genes, such as the human growth hormone mini-gene as a polyadenylation sequence and recently reported in beta cells using the mouse insulin promoter to drive Cre expression [2]. Conversely, Cre expression driven by the myosin heavy chain- $\alpha$ promoter to generate cardiac myocytespecific gene KOs produces a transient heart failure phenotype [3]. These studies highlight important considerations to be taken into account when using Cre-loxP systems to study gene/protein function, in particular the use of the appropriate control littermate for comparison. Here, we report whether similar considerations apply to the tamoxifen-inducible human $\alpha$ skeletal actin (HSA) promoter to drive Cre expression in skeletal muscle.

\section{Methods}

Animal care All animals received care according to the Canadian Council on Animal Care and all procedures were approved by the University of Alberta Health Sciences Animal Welfare Committee. All animal husbandry was performed by University of Alberta Health Sciences Laboratory Animal Services staff. Six-week-old HSA-Cre male mice [Jackson Laboratory, Bar Harbor, ME, USA; stock no. 025750; $\operatorname{Tg}$ (ACTA1-cre/Esr1*)2Kesr] and their wild-type (WT) littermates (Jackson Laboratory, stock no. 000664, C57BL/6J) received daily i.p. injections with either vehicle (corn oil) or tamoxifen $(50 \mathrm{mg} / \mathrm{kg}$ ) for 5 days. All mice were allowed a 1 week washout post-tamoxifen, following which 8week-old mice were placed on either a low-fat diet (LFD) (10\% kJ from lard; Research Diets, New Brunswick, NJ, USA, D12450J) or a high-fat diet (HFD) $(60 \% \mathrm{~kJ}$ from lard; Research Diets D12492) for 12 weeks. Animals were euthanised at 20 weeks of age via i.p. injection of sodium pentobarbital $(12 \mathrm{mg}$ ) following a $2 \mathrm{~h}$ fast. No randomisation was carried out and experimenters were not blinded to group assignment and outcome assessment.

Cell culture $\mathrm{C} 2 \mathrm{C} 12$ skeletal muscle myoblasts (ATCC, Rockville, MD, USA) were cultured and differentiated into myotubes as previously described [4]. Following 4 days of 
differentiation, C2C12 myotubes underwent Lipofectaminemediated transfection with either a control or Cre recombinase-expressing plasmid (Addgene, Cambridge, MA, USA, plasmid no. 13775) for $24 \mathrm{~h}$. Media was replaced with serum-free media for $24 \mathrm{~h}$ and collected. INS-1 832/3 cells (referred to, herein, as INS-1) were cultured and used for the assessment of glucose-stimulated insulin secretion (GSIS) as previously described [5]. As a positive control for stimulating GSIS, separate groups of INS-1 cells were treated with D-Ala ${ }^{2}$ glucose-dependent insulinotropic polypeptide (D-Ala $\left.{ }^{2} \mathrm{GIP}\right)$.

Assessment of glucose homeostasis I.p. glucose ( $2 \mathrm{~g} / \mathrm{kg}$ body weight) and insulin $(0.7 \mathrm{U} / \mathrm{kg}$ body weight) tolerance tests were performed in 16- and 17-week-old overnight-fasted WT and HSA-Cre mice, respectively. Blood glucose measurements were performed via tail bleed with the Accu-Chek Advantage system (Roche, Indianapolis, IN, USA) in blood samples obtained at the end of the fast ( $0 \mathrm{~min})$, followed by blood samples at 15, 30, 60, 90 and $120 \mathrm{~min}$ following glucose/insulin administration. Plasma was extracted from whole blood collected from mouse tails for measuring insulin levels as previously described [6].

Whole-body in vivo metabolic assessment Indirect calorimetry was performed in 18-week-old WT or HSA-Cre mice using Oxymax metabolic cages (Columbus Instruments, Columbus, OH, USA). Animals were initially acclimatised in the system for $24 \mathrm{~h}$, following which the subsequent $24 \mathrm{~h}$ was used for data collection (e.g. oxygen consumption) as previously described [6].

Determination of muscle triacylglycerol (TAG) levels Frozen powdered gastrocnemius muscle tissue was extracted in a 2:1 chloroform:methanol solution, following which the supernatant phase was retained for the assessment of TAG content with an enzymatic assay kit (Wako Pure Chemical Industries, Osaka, Japan) as previously described [6].

Western blotting Protein samples were prepared from powdered frozen gastrocnemius tissue $(20 \mathrm{mg})$ homogenised in protein lysis buffer, following which they were subjected to western blotting protocols as previously described [7]. Akt and phospho (p)-Akt (9272S and 4060L); glycogen synthase kinase (GSK) $3 \beta$ and p-GSK3 $\beta$ (5676S and 9331L); mammalian target of rapamycin (mTOR) and p-mTOR (2983S and 5536); and Cre recombinase (15036) antibodies were purchased from Cell Signaling (Danvers, MA, USA) and prepared in a 1:500 dilution in 5\% bovine serum albumin (BSA).

Real-time PCR analysis First-strand cDNA was synthesised from total RNA using the SuperScript III synthesis system (Invitrogen, Carlsbad, CA, USA). Real-time PCR was subsequently carried out using TaqMan gene expression assays
(Applied Biosystems, Foster City, CA, USA) for quantification of relative mRNA transcript levels (see electronic supplementary material [ESM] Table 1 for primer list) as previously described [7].

Immunohistochemistry Pancreases were fixed in 4\% formalin and embedded in paraffin, sliced $(5 \mu \mathrm{m})$ and mounted on slides. Citrate buffer was used for antigen retrieval, and immunohistochemistry was performed using either guinea pig anti-insulin antibody (Abcam, Cambridge, MA, USA) at a 1:250 dilution or rabbit anti-glucagon antibody (Millipore, Billerica, MA, USA) at a 1:5000 dilution in 5\% BSA. Antibodies were visualised using the 3,3'-diaminobenzidine colorimetric system and images were captured using a Leica DMRE microscope (Leica, Wetzlar, Germany) as previously described [8].

Nuclear/cytoplasmic fractionation Nuclear and cytoplasmic protein fractions from gastrocnemius tissue were extracted using the Nuclear Extract Kit (Active Motif, Carlsbad, CA) according to the manufacturer's protocol as previously described [9]. For western blotting analyses, voltage-dependent anion-selective channel (VDAC)1 and histone H3 were used as cytoplasmic and nuclear markers, respectively.

Co-immunoprecipitation Equal amounts of protein lysates were incubated with anti-Cre antibody and a non-specific IgG antibody as a negative control overnight at $4{ }^{\circ} \mathrm{C}$. To pull down the antigen-antibody complex, $40 \mu$ protein $G$ Dynabeads (Thermo Fisher Scientific, Waltham, MA, USA) were added to all samples and incubated at $4^{\circ} \mathrm{C}$ for $2 \mathrm{~h}$. The beads were washed three times with ice-cold PBS, and bound proteins were eluted by boiling at $95^{\circ} \mathrm{C}$ for $5 \mathrm{~min}$ with $20 \mu \mathrm{l}$ of SDS protein loading buffer, following which they were subsequently processed for western blotting as previously described [9].

Statistical analysis No samples were excluded from our data analyses. All values are presented as means \pm SEM. Significant differences were determined using an unpaired, two-tailed Student's $t$ test or one- or two-way ANOVA followed by Bonferroni post hoc analysis.

\section{Results}

Glucose tolerance is improved in HSA-Cre mice We observed no differences in body weight or composition between WT and HSA-Cre mice fed either a LFD or HFD for 12 weeks (Fig. 1a, b). However, while HSA-Cre mice fed a LFD demonstrated no change in glucose tolerance and circulating insulin levels, robust improvements in both variables were observed in HFD-fed HSA-Cre mice (Fig. 1c, d). 
a

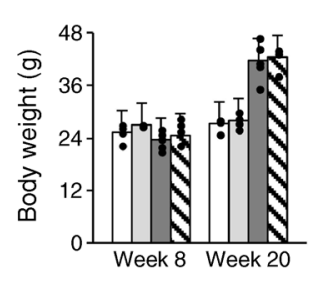

e

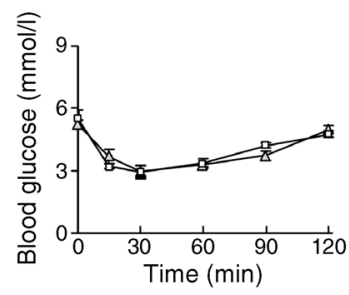

i

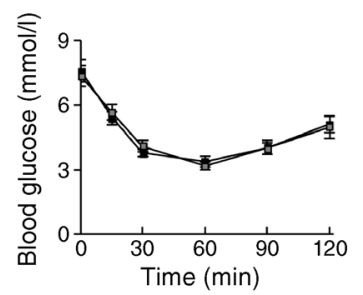

b

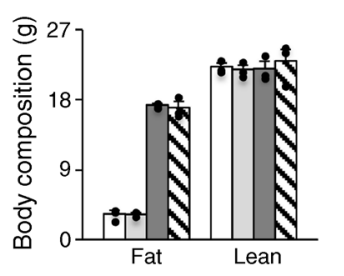

f
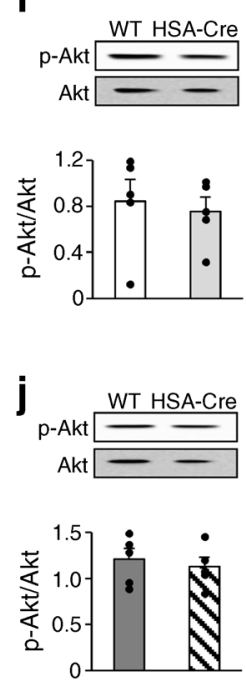

C

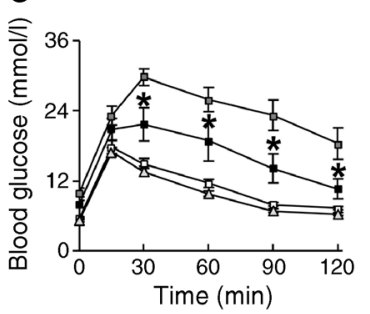

g
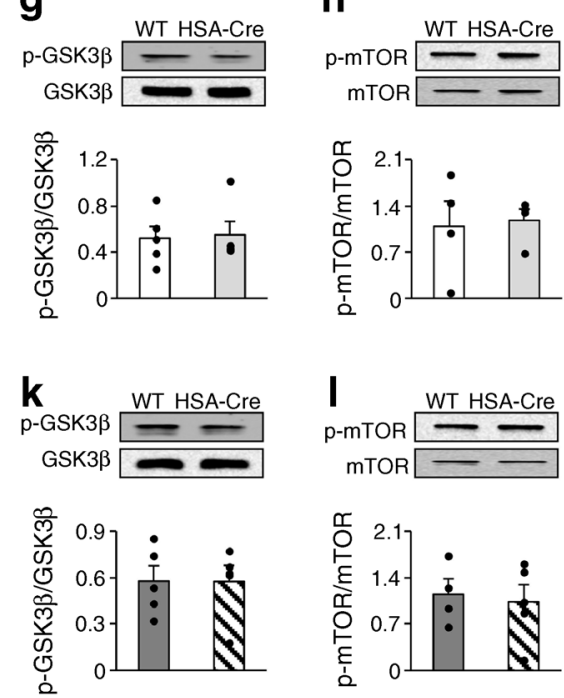

h
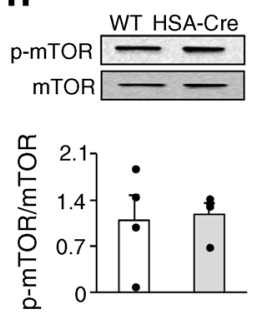

d

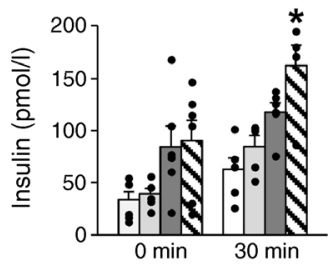

$\mathbf{m}$

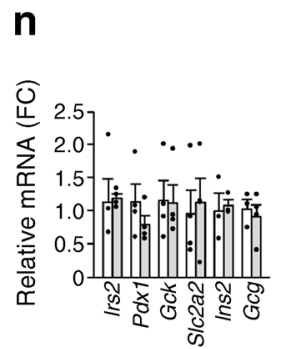

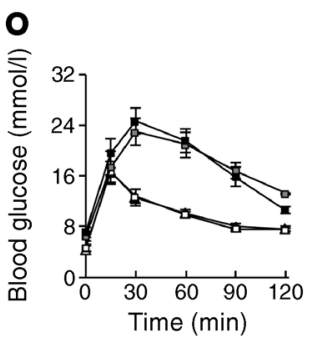

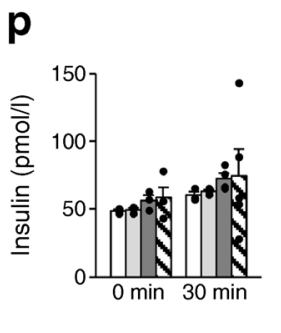

Fig. 1 Improved glucose tolerance in HSA-Cre mice. (a) Body weight $(n=6)$, (b) body composition $(n=3)$, (c) glucose tolerance $(n=13)$ and (d) circulating insulin levels $(n=5-6)$ in tamoxifen-treated WT and HSACre mice supplemented with either a LFD or HFD for 8 weeks. (e, i) Insulin tolerance $(\mathbf{e}, n=5 ; \mathbf{i}, n=9)$ and $(\mathbf{f}-\mathbf{h}, \mathbf{j}-\mathbf{l})$ insulin signalling (Akt, GSK $3 \beta$ and mTOR phosphorylation) in gastrocnemius muscle $(n=4-5)$ from tamoxifen-treated WT and HSA-Cre mice supplemented with a LFD $(\mathbf{e}-\mathbf{h})$ or HFD (i-l) for 9 weeks. (m) Insulin and glucagon staining of pancreatic islets from tamoxifen-treated WT and HSA-Cre mice supplemented with a LFD $(n=4)$. Scale bars, $10 \mu \mathrm{m}$. (n) mRNA profiling of key genes regulating pancreatic function in pancreases from tamoxifen-

Insulin tolerance and signalling are not changed in HSA-Cre mice WT and HSA-Cre mice fed a LFD exhibited similar insulin sensitivity during an i.p. insulin tolerance test (Fig. 1e), whereas phosphorylation of canonical members of the insulin signalling pathway (Akt, GSK $3 \beta$, mTOR) was similar in gastrocnemius muscles following a $2 \mathrm{~h}$ fast (Fig. 1fh). These indices of insulin sensitivity were also similar between WT and HSA-Cre mice fed a HFD (Fig. 1i-1). treated WT and HSA-Cre mice supplemented with a LFD $(n=3-4)$. FC, fold change. (o) Glucose tolerance $(n=6)$ and (p) circulating insulin levels $(n=3-5)$ in corn-oil-treated WT and HSA-Cre mice supplemented with either a LFD or HFD for 8 weeks. (q) Insulin tolerance in corn-oiltreated WT and HSA-Cre mice supplemented with either a LFD or HFD for 9 weeks $(n=6)$. White bars/squares, LFD WT mice; light-grey bars/ triangles, LFD HSA-Cre mice; dark-grey bars/squares, HFD WT mice; hatched bars/black squares, HFD HSA-Cre mice. Values are means \pm SEM. Differences were determined using two-way ANOVA, followed by Bonferroni post hoc analysis. $* p<0.05$ vs WT counterpart

Importantly, insulin and glucagon staining-based immunohistochemistry revealed no discernible differences in islet morphology, whereas mRNA expression of key genes regulating pancreatic function was also similar between tamoxifen-treated WT and HSA-Cre mice (Fig. 1m, n). In addition, we could not detect mRNA expression for Cre recombinase in pancreatic (islet) tissues of HSA-Cre mice (data not shown). 
Glucose tolerance in the absence of tamoxifen-mediated liberation of Cre recombinase To demonstrate that the improved glucose tolerance in HSA-Cre mice is dependent on tamoxifen-mediated liberation of Cre recombinase within skeletal muscle, we repeated our studies in mice treated with vehicle (corn oil). Corn-oil-treated HSA-Cre mice fed a LFD or HFD demonstrated no differences in glucose tolerance, displayed similar circulating insulin levels in response to glucose and had similar insulin sensitivity compared with their WT littermates (Fig. 1o-q).

Skeletal muscle-specific Cre recombinase expression Western blotting experiments demonstrated that Cre recombinase is expressed in both corn-oil- and tamoxifen-treated HSA-Cre skeletal muscles (gastrocnemius and quadriceps), but not the liver or heart (Fig. 2a). Because Cre recombinase in the HSACre mouse is bound to a complex with a mutated oestrogen receptor and heat shock protein (Hsp)90, we next demonstrated, using co-immunoprecipitation, that Cre recombinase was still associated with Hsp90 in corn-oil-treated HSA-Cre mice, whereas no association between Cre recombinase and Hsp90 was observed following treatment with tamoxifen (Fig. 2b). Likewise, tamoxifen treatment of HSA-Cre mice resulted in the nuclear translocation of Cre recombinase (Fig. 2c).

\section{Substrate preference and energy expenditure in HSA-Cre} mice Indirect calorimetry studies in tamoxifen-treated, HFDfed mice at 18 weeks of age showed similar respiratory exchange ratios, whole-body oxygen consumption rates, ambulatory activity, and water and food intake between genotypes (Fig. $3 \mathrm{a}-\mathrm{e})$. Following euthanasia of mice, we assessed TAG content in gastrocnemius muscle and $\mathrm{mRNA}$ expression profiles for key regulators of energy metabolism/mitochondrial function, since improved mitochondrial function often elicits improvements in glucose homeostasis [10]. However, we observed no differences in gastrocnemius muscle TAG content between WT and HSACre mice (Fig. 3f), nor did we observe changes in mRNA expression for a number of genes involved in mitochondrial function (Fig. 3g), fatty acid metabolism/oxidation (Fig. 3h, i) or ketone body metabolism/oxidation (Fig. 3j). Conversely, nonsignificant increases in the genes encoding peroxisome proliferator-activated receptor (PPAR)- $\delta$ and PPAR $\gamma$ coactivator $1-\alpha$ expression were observed in HSA-Cre mice (Fig. 3i; $p=0.069$ and 0.068 , respectively).

\section{Conditioned media (CM) from C2C12 myotubes overexpress- ing Cre recombinase does not augment insulin secretion We} posited that expression/activation of Cre recombinase in skeletal muscle leads to the production of a secreted factor that improves beta cell insulin secretion in HSA-Cre mice. Accordingly, we transfected Cre recombinase into $\mathrm{C} 2 \mathrm{C} 12$ myotubes (Fig. 3k), following which CM from these cells was applied to INS-1 beta cells during a GSIS test. CM from
C2C12 myotubes overexpressing Cre recombinase failed to augment GSIS compared with CM from C2C12 myotubes transfected with a control plasmid, whereas D-Ala ${ }^{2}$ GIP robustly enhanced GSIS (Fig. 31).

\section{Discussion}

Our findings support the notion that tissue-specific Cre recombinase expression may influence cellular/tissue function, as we demonstrate for the first time that expression/ activation of Cre recombinase in skeletal muscle improves glucose tolerance. It is possible that Cre recombinase expression may not be truly confined to skeletal muscle in HSA-Cre mice, potentially explaining our unexpected findings, yet Cre recombinase was undetectable in the heart, liver and pancreas of HSA-Cre mice, ruling against leaky expression as a mechanism. The observed improvements were not related to changes in insulin sensitivity/signalling, but rather to increases in circulating insulin. Moreover, insulin levels only showed differences in tamoxifen-treated HFD-fed HSA-Cre mice during an i.p. glucose tolerance test, as fasted (Fig. 1d) and ad libitum (data not shown) insulin levels were identical between tamoxifen-treated HFD-fed HSA-Cre mice and their WT littermates. It remains unclear how Cre recombinase expression in skeletal muscle impacts insulin secretion, as our CM experiments in $\mathrm{C} 2 \mathrm{C} 12$ myotubes overexpressing Cre recombinase failed to augment GSIS from INS-1 cells. Nonetheless, it is possible that Cre recombinase in skeletal muscle influences secreted factors to a different extent than that in $\mathrm{C} 2 \mathrm{C} 12$ myotubes transfected with a Cre recombinase plasmid. Hence, further work is necessary to determine the mechanism(s) by which skeletal muscle Cre recombinase expression influences circulating insulin levels and/or insulin secretion. Our study was only conducted in male mice, and it remains to be seen whether female HSA-Cre mice exhibit the same phenotype.

Our observations have relevance as Cre-loxP systems are often used to identify how specific genes/proteins in skeletal muscle impact glycaemia and insulin sensitivity in experimental models of obesity. However, the vast majority of studies using Cre-loxP systems to investigate glucose homeostasis and insulin resistance have used the floxed mouse as the control littermate. Reasons for this likely include the rationale that a mouse expressing a floxed gene may produce a phenotype itself, whereas breeding strategies designed to produce both tissue-specific Cre recombinase control littermates and the corresponding tissue-specific gene $\mathrm{KO}$ are much more complex and met with a reduced frequency of required genotypes. Examples where ramifications may have been missed through the use of inappropriate control littermates to study musclespecific actions include a report that used muscle creatine kinase (MCK)-Cre mice to generate mice deficient in 
a
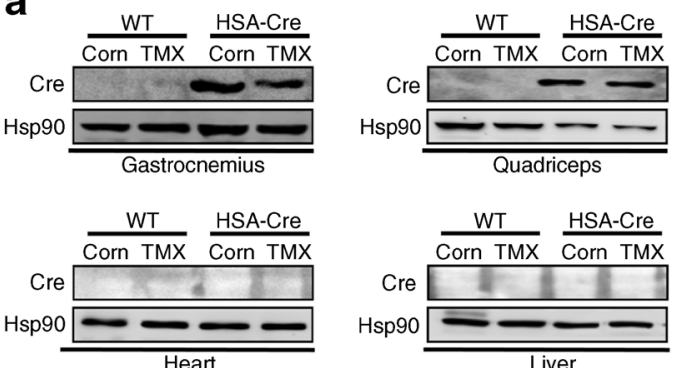

Fig. 2 Cre recombinase expression and nuclear translocation in HSA-Cre mice. (a) Cre recombinase expression in gastrocnemius muscle, quadriceps muscle, heart and liver from WT and HSA-Cre mice treated with corn oil (Corn) or tamoxifen (TMX) $(n=4)$. (b) Co-immunoprecipitation (IP) demonstrating that Cre recombinase remained bound to Hsp90 in HSACre mice treated with corn oil, whereas TMX treatment liberated Cre

muscle-specific carnitine palmitoyltransferase-1b [11], and another that used the non-inducible HSA-Cre mouse to generate mice deficient in muscle-specific growth factor receptorbound protein 10 [12]. Although these studies demonstrated improvements in glycaemia during obesity [11] and ageing
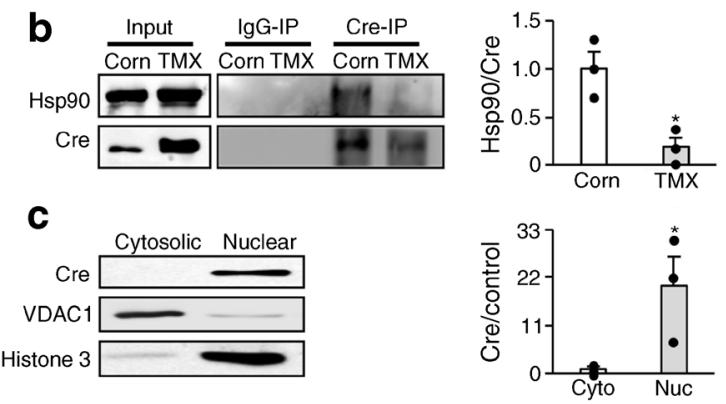

recombinase from its mutated oestrogen receptor complex with Hsp90 $(n=3)$. (c) Nuclear and cytosolic fractions were prepared from gastrocnemius muscles from TMX-treated HSA-Cre mice, and the presence of Cre recombinase was seen only within the nuclear fraction $(n=3)$. Cre was normalised to VDAC1 (cytosol [cyto]) or histone 3 (nuclear [Nuc]). Values are means \pm SEM. * $p<0.05$, unpaired, two-tailed Student's $t$ test

[12], the respective floxed mouse was used as the control littermate. Hence, the authors' observations could potentially be due to the expression of Cre within skeletal muscle. It is also unknown whether MCK-Cre or non-inducible HSA-Cre mice exhibit a similar phenotype to the tamoxifen-inducible
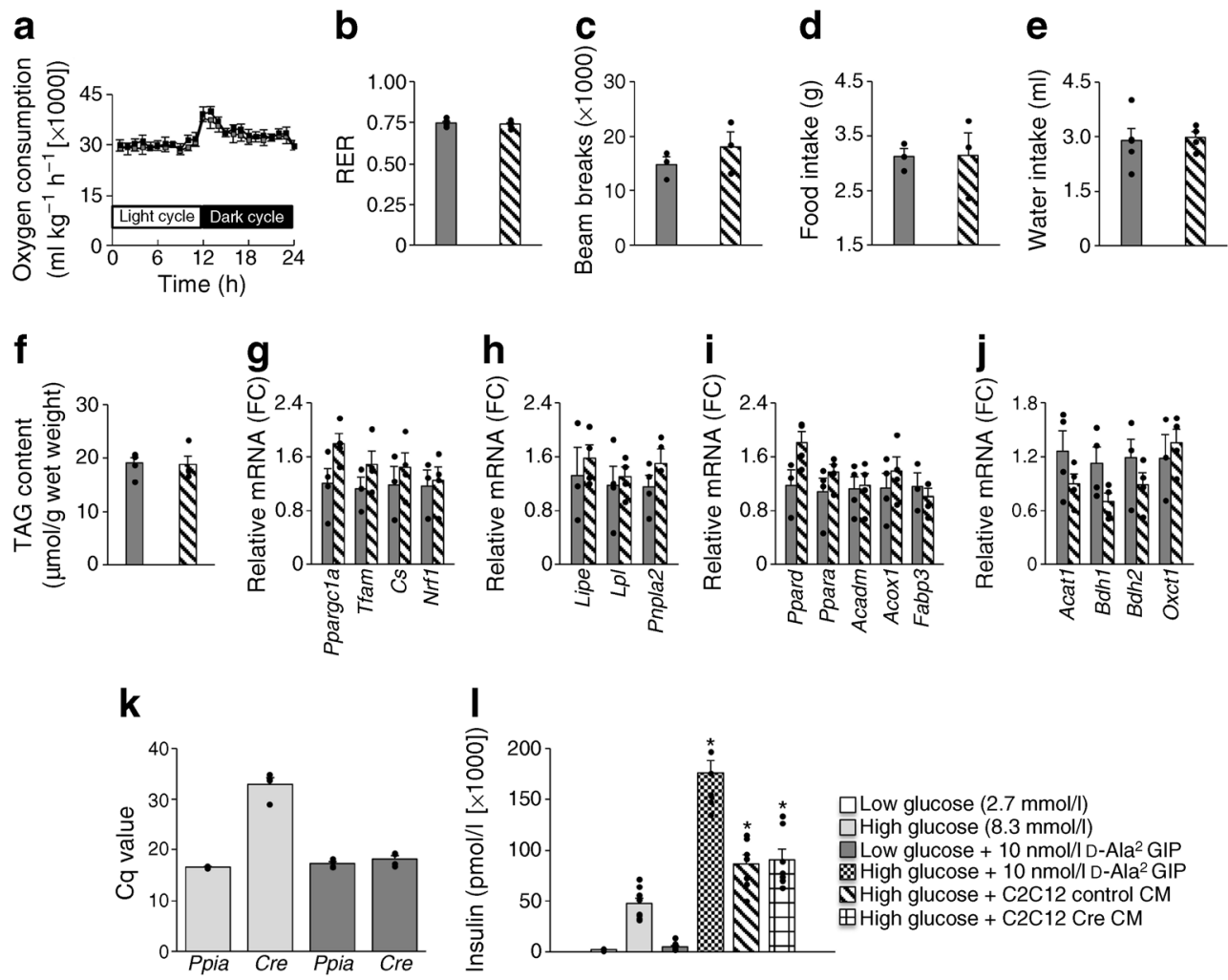

Fig. 3 Characterisation of potential contributors to improved glucose tolerance in HSA-Cre mice. (a) Whole-body oxygen consumption rate $(n=5),(\mathbf{b})$ respiratory exchange ratio (RER) $(n=5)$, (c) ambulatory activity $(n=3)$, (d) food intake $(n=3)$ and (e) water intake $(n=4)$ in tamoxifen-treated WT and HSA-Cre mice supplemented with a HFD for 10 weeks. (f) Gastrocnemius muscle TAG content in tamoxifen-treated WT and HSA-Cre mice supplemented with a HFD for 12 weeks $(n=4$ 5). mRNA expression profiles for genes involved in (g) mitochondrial function, (h) lipolysis, (i) fatty acid oxidation and (j) ketone body metabolism in tamoxifen-treated WT and HSA-Cre mice supplemented with a

HFD for 12 weeks $(n=4)$. Dark-grey bars/squares, HFD WT mice; hatched bars/black squares, HFD HSA-Cre mice. (k) Cre recombinase mRNA expression in $\mathrm{C} 2 \mathrm{C} 12$ myotubes transfected with a control plasmid (light-grey bars) or a Cre recombinase-expressing plasmid construct (dark-grey bars) $(n=4)$. (I) Insulin secretion in INS-1 cells treated with $\mathrm{CM}$ from $\mathrm{C} 2 \mathrm{C} 12$ myotubes overexpressing Cre recombinase $(n=7-8)$. Values are means \pm SEM. $* p<0.05$ vs the high-glucose only group, oneway ANOVA followed by Bonferroni post hoc analysis. Cq, quantification cycle; FC, fold change 
HSA-Cre mouse, and it will be important for future studies to determine if our results translate to these models.

Taken together, it appears that the HSA-Cre mouse is a necessary control littermate for any study using a Cre-loxP system to investigate skeletal muscle gene/protein function in the experimental setting of obesity/insulin resistance. Accordingly, any study using the floxed mouse as the sole control littermate will be unable to conclude whether an improvement in glucose tolerance in the corresponding skeletal muscle-specific gene $\mathrm{KO}$ is due to deletion of the gene within muscle itself, or the result of Cre recombinase activity.

Acknowledgements We thank J. Kruger (Health Sciences Laboratory Animal Services, University of Alberta) for husbandry and maintenance of our HSA-Cre mouse colony.

Data availability The datasets generated and presented in the current study are available from the corresponding author on reasonable request.

Funding These studies were supported in part by a discovery grant (RGPIN 04946) from the Natural Sciences and Engineering Research Council of Canada to JRU. JRU is a Scholar of the Canadian Diabetes Association and a New Investigator of the Heart and Stroke Foundation of Alberta, NWT \& Nunavut. RAB is a postdoctoral fellow of the Canadian Institutes of Health Research and the Canadian Diabetes Association.

Duality of interest The authors declare that there is no duality of interest associated with this manuscript.

Contribution statement RAB, JEC and JRU were involved with conception and design of the study. RAB, KG, MDM, KLH, MA, HA and FE were involved with data acquisition. $\mathrm{RAB}, \mathrm{KG}$ and MDM were involved with data analysis and interpretation. RAB and JRU drafted the manuscript. All authors contributed to critically revising the article for important intellectual content and gave their final approval of the version to be published. JRU is the guarantor of this work.

\section{References}

1. Glaser S, Anastassiadis K, Stewart AF (2005) Current issues in mouse genome engineering. Nat Genet 37:1187-1193

2. Oropeza D, Jouvet N, Budry L et al (2015) Phenotypic characterization of MIP-CreERT1Lphi mice with transgene-driven islet expression of human growth hormone. Diabetes 64:3798-3807

3. Koitabashi N, Bedja D, Zaiman AL et al (2009) Avoidance of transient cardiomyopathy in cardiomyocyte-targeted tamoxifen-induced MerCreMer gene deletion models. Circ Res 105:12-15

4. Ussher JR, Jaswal JS, Wagg CS et al (2009) Role of the atypical protein kinase Czeta in regulation of 5'-AMP-activated protein kinase in cardiac and skeletal muscle. Am J Physiol Endocrinol Metab 297:E349-E357

5. Hohmeier HE, Mulder H, Chen G, Henkel-Rieger R, Prentki M, Newgard CB (2000) Isolation of INS-1-derived cell lines with robust ATP-sensitive $\mathrm{K}+$ channel-dependent and -independent glucose-stimulated insulin secretion. Diabetes 49:424-430

6. Ussher JR, Fillmore N, Keung W et al (2016) Genetic and pharmacological inhibition of malonyl CoA decarboxylase does not exacerbate age-related insulin resistance in mice. Diabetes 65:1883-1891

7. Ussher JR, Baggio LL, Campbell JE et al (2014) Inactivation of the cardiomyocyte glucagon-like peptide-1 receptor (GLP-1R) unmasks cardiomyocyte-independent GLP-1R-mediated cardioprotection. Mol Metab 3:507-517

8. Kitamura T, Kitamura Y, Nakae J et al (2004) Mosaic analysis of insulin receptor function. J Clin Invest 113:209-219

9. Gopal K, Saleme B, Al Batran R et al (2017) FoxO1 regulates myocardial glucose oxidation rates via transcriptional control of pyruvate dehydrogenase kinase 4 expression. Am J Physiol Heart Circ Physiol 313:H479-H490

10. Muoio DM, Neufer PD (2012) Lipid-induced mitochondrial stress and insulin action in muscle. Cell Metab 15:595-605

11. Vandanmagsar B, Warfel JD, Wicks SE et al (2016) Impaired mitochondrial fat oxidation induces FGF21 in muscle. Cell Rep 15: 1686-1699

12. Holt LJ, Brandon AE, Small L et al (2018) Ablation of Grb10 specifically in muscle impacts muscle size and glucose metabolism in mice. Endocrinology 159:1339-1351 